\title{
Computerized Tomographic Assessment of Vocal Cord Palsy: Otolaryngologist's Purview
}

\author{
${ }^{1}$ Apoorva K Pandey, ${ }^{2}$ Sharad Gangrade, ${ }^{3}$ Ankur Malhotra, ${ }^{4}$ Arvind Varma, ${ }^{5}$ Tripti Maithani
}

\begin{abstract}
Aims and objectives: Vocal cord palsy (VCP) can be definitely depicted on computed tomograpy (CT) scan by identifying key points at the level of true vocal cords and aryepiglottic folds. There are various VCP mimics and imaging shortcomings, and these can usually be circumvented by thoughtfully assessing the scan plan and level, and a diligent search for additional characteristics.
\end{abstract}

Materials and methods: This retrospective study consisted of 10 cases of unilateral VCP, who all had been clinically diagnosed of VCP and further evaluated by CT. Pre- and postcontrast enhanced axial CT from skull base to aorto-pulmonary window were done and multiplanar three-dimensional reconstruction of images in coronal and sagittal planes along with volumerendered imaging was done.

Results: Most consistent findings in VCP were thickening of ipsilateral aryepiglottic fold and medialization of ipsilateral aryepiglottic fold $(100 \%)$, followed by dilation of ipsilateral pyriform sinus $(90 \%)$ and dilatation of ipsilateral laryngeal ventricle $(80 \%)$. Etiologic causes were ascertained in three cases: Thyroid malignancy, tuberculosis, and pyriform sinus malignancy. The last etiology was probably a VCP mimic.

Conclusion: Radiologic evaluation is inarguably useful for determining the etiology of VCP, particularly for lesions within neck and thoracic cavity. Most crucial objective in assessing a case of VCP is to exclude the presence of a life-threatening primary lesion as the cause of VCP.

Keywords: Chest radiograph, Computed tomography, Idiopathic, Recurrent laryngeal nerve, Superior laryngeal nerve, Vocal cord palsy.

How to cite this article: Pandey AK, Gangrade S, Malhotra A, Varma A, Maithani T. Computerized Tomographic Assessment of Vocal Cord Palsy: Otolaryngologist's Purview. Int J Phonosurg Laryngol 2016;6(2):57-63.

Source of support: Nil

Conflict of interest: None

${ }^{1,5}$ Professor, ${ }^{2}$ Senior Resident, ${ }^{3}$ Assistant Professor, ${ }^{4}$ Associate Professor

1,4,5 Department of ENT, Sri Guru Ram Rai Institute of Medical and Health Sciences, Dehradun, Uttarakhand, India

${ }^{2,3}$ Department of Radiodiagnosis, Sri Guru Ram Rai Institute of Medical and Health Sciences, Dehradun, Uttarakhand, India

Corresponding Author: Apoorva K Pandey, Professor Department of ENT, Sri Guru Ram Rai Institute of Medical and Health Sciences, Dehradun, Uttarakhand, India, Phone: +911352760439, e-mail: apoorva.pande@yahoo.co.in

\section{INTRODUCTION}

Vocal cord paralysis (VCP) can be caused by various pathologies in neck and mediastinum, including neoplastic, inflammatory, vascular, traumatic, congenital, and idiopathic conditions, and may be the only presenting sign of underlying pathology. Vocal cord palsy can be caused by any pathology affecting the vagus nerve in its course above the branching of the recurrent laryngeal nerve (RLN) or due to involvement of RLN per se. Vocal cord paralysis most commonly affects one side and can also be bilateral. Symptoms of VCP include: Hoarseness of voice, vocal fatigue, loss of vocal pitch, shortness of breath, and aspiration. ${ }^{1}$ In almost half of all cases of $\mathrm{VCP}$, who are essentially idiopathic, sectional imaging may fail to detect a lesion along the course of either vagus nerve or the RLN. In these cases, imaging differentiation of a central vagal lesion from a RLN lesion can be discernible by observing the effects of a pharyngeal plexus involvement on the ipsilateral pharyngeal constrictor muscles. ${ }^{2}$ Nevertheless, up to $40 \%$ of cases with VCP may be asymptomatic, ${ }^{3}$ so in these cases imaging studies can reliably suggest presence of VCP on routine neck scanning without a history suggestive of hoarseness or cord paralysis. ${ }^{2}$ Regardless of idiopathic etiology of reasonable number of cases, imaging techniques have an important role in investigating etiologic cases. Careful assessment of findings on computed tomography (CT) evaluation, including skull base to upper mediastinum, can be indispensable in (a) directing timely specialist referral, (b) recommending further necessary imaging, and (c) deciding appropriate surgical, medical, or watchful waiting strategies. ${ }^{4}$

This clinical study depicts the imaging evaluation of the possible location/site or extent of vagal or RLN lesion causing VCP and imaging characteristics and mimics of VCP in 10 cases during an 18-month duration at a tertiary care center.

\section{MATERIALS AND METHODS}

This retrospective study comprised 10 cases of clinically established VCP cases (unilateral/bilateral), who presented at the Otorhinolaryngology Department of Tertiary Care Centre and were subsequently evaluated by the CT scan in Radiology Department from January 2015 to June 2016. A 
complete history, with complaints of hoarseness of voice, cough, aspiration, etc., was noted. Sex and age distribution was also included. A thorough ENT examination was done along with cranial nerve evaluation and endoscopic documentation of VCP. Routine blood investigations and chest radiograph were also done in almost all cases. All studies were performed on multislice Philips ingenuity core 128-slice CT machine. The CT scan in a patient was performed as pre- and postcontrast-enhanced study from skull base to aorto-pulmonary window. The CT scan parameters were contiguous 3-mm axial scans with $0.9 \mathrm{~mm}$ reconstruction, pitch 0.45 , and field of view (FOV) $250 \mathrm{~mm}$. All radiologic findings were further correlated with laryngoscopic findings.

Only eight imaging signs of VPC, in accordance with criteria as laid by Landman, were evaluated after multiplanar three-dimensional reconstruction of images in coronal and sagittal planes with volume-rendered images. ${ }^{5}$ Signs evaluated were: (1) Thickening of ipsilateral aryepiglottic fold, (2) medialization of ipsilateral aryepiglottic fold, (3) dilatation of ipsilateral pyriform sinus, (4) dilatation of ipsilateral laryngeal ventricle, (5) dilatation of ipsilateral vallecula, (6) fullness of contralateral vocal cord, (7) anteromedial displacement of ipsilateral arytenoid cartilage, and (8) flattening of subglottic arch demonstrated in coronal reconstruction. Radiological evaluation and confirmation of the affected side was also correlated with clinical findings. Evaluation was also done to detect the possible site or location of the vagal or RLN lesion. Every case was also analyzed to confirm the presence of

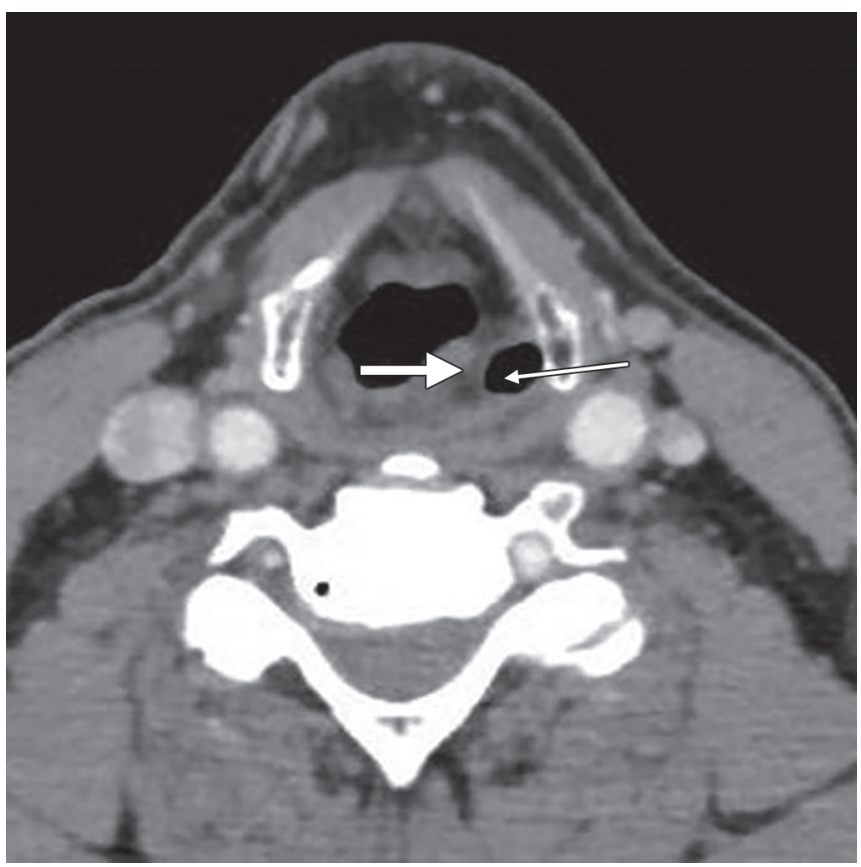

Fig. 1: Left RLN palsy. Axial CT scan obtained at the level of the hypopharynx shows dilatation of the ipsilateral pyriform fossa with air (thin arrow) and the medially rotated, thickened ipsilateral aryepiglottic fold (thick arrow) imaging signs for pharyngeal involvement, suggestive of central or high vagal lesion.

\section{RESULTS}

In our study, comprising 10 cases, the youngest patient was 40 years and oldest was 75 years, with mean age 62.9 years. Most patients were elderly in 5 th to 7 th decade of life. Male to female ratio was 4:1. Unilateral VCP was observed in 9 cases (90\%), and 1 case (10\%) was of bilateral VCP. Of total 10 VCP cases, 7 cases (70\%) were left-sided VCP and 2 cases (20\%) were of right-sided VCP.

On specific imaging findings in 9 patients with unilateral VCP, ipsilateral thickening and medialization of aryepiglottic fold were observed in 9 (100\%) patients, ipsilateral dilatation of pyriform sinus was observed in $8(88.8 \%)$ patients (Fig. 1), ipsilateral laryngeal ventricle dilatation in $7(77.7 \%)$ patients, ipsilateral anteromedial displacement of arytenoid cartilage in $4(44.4 \%)$ patients, ipsilateral subglottic fullness (Fig. 2) in 3 (33.3\%) patients, ipsilateral dilatation of vallecula in $4(44.4 \%)$ patients, and fullness of ipsilateral pharyngeal wall (Fig. 3) in 2 (22.2\%) patients. In patient with bilateral VCP, there were thickening and medialization of bilateral aryepiglottic fold with dilatation of bilateral pyriform sinus and laryngeal ventricle. There was dilatation of left vallecula noted on left side only (Table 1).

Of the 9 patients with unilateral VCP, we observed consistently two signs in all $9(100 \%)$ patients: (1) Ipsilateral thickening and (2) medialization of aryepiglottic

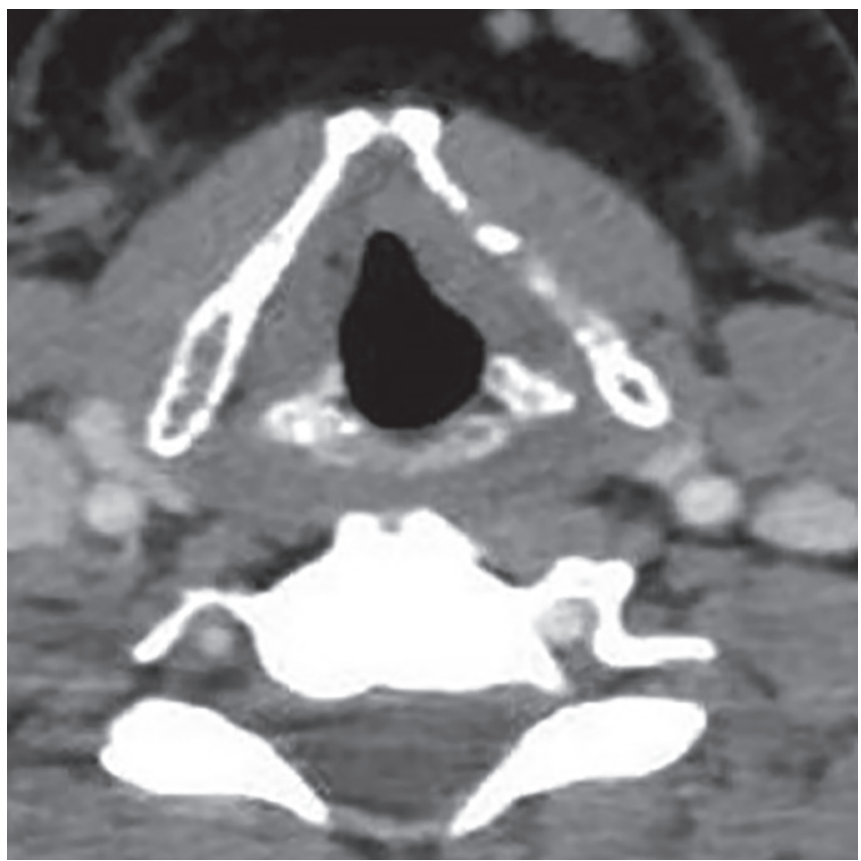

Fig. 2: Patient with right VCP. Axial image showing fullness of subglottic region 


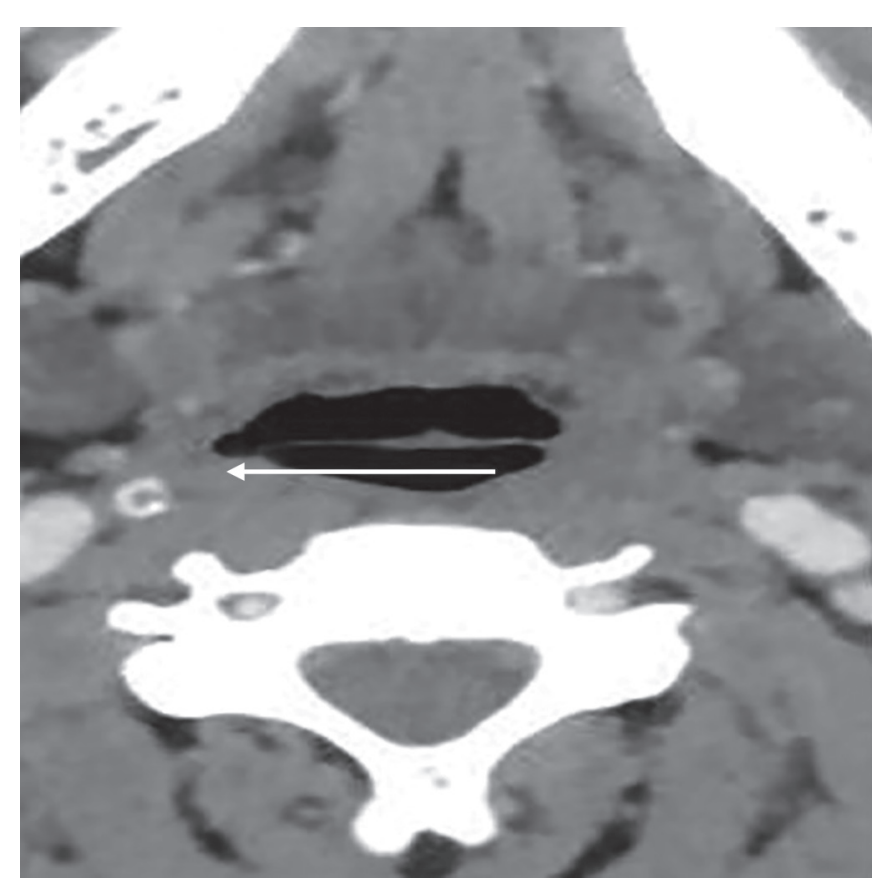

Fig. 3: Patient with right VCP. Axial image showing fullness of ipsilateral pharyngeal wall (thin arrow)

fold (Fig. 4). Ipsilateral dilatation of pyriform sinus was noted in 8 cases $(88.8 \%)$, and ipsilateral laryngeal ventricle dilatation was seen in 7 cases $(77.77 \%)$ (Fig. 5). In one patient of unilateral VCP where no ipsilateral pyriform sinus dilatation was seen, but ipsilateral laryngeal ventricle dilatation was noted, while in other 2 unilateral VCP patients with no ipsilateral laryngeal ventricle dilatation, ipsilateral pyriform sinus dilatation was observed.

Pathological lesions were observed in three patients where thyroid mass in first case and pyriform sinus growth in another patient were identified. Thyroid mass came out to be malignancy on investigation and pyriform malignancy was later histologically proved. In almost all cases of unilateral VCP, radiologic findings correlated well with

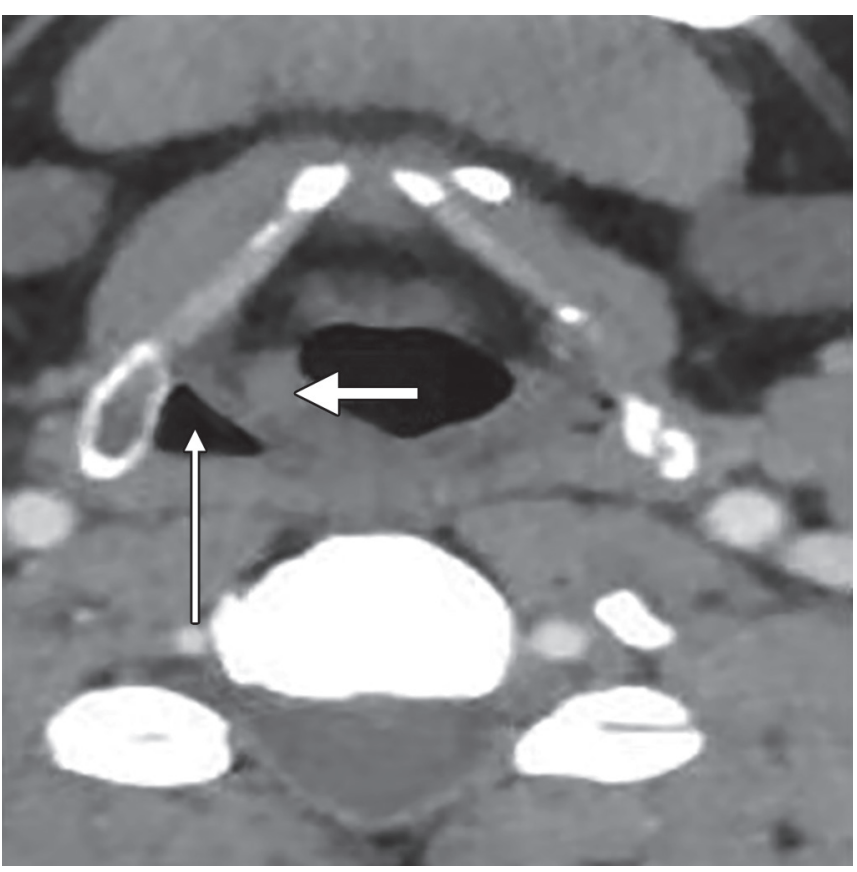

Fig. 4: Patient with right VCP. Axial image shows thickened and medially rotated aryepiglottic fold (thick arrow) with ipsilateral pyriform sinus dilatation (thin arrow)

the clinical diagnosis except in pyriform sinus malignancy cases, where on direct laryngoscopy VC was found to be fixed, rendering this case to be a possible VCP mimic. In third patient, heterogeneously enhancing cervical lymph node on left side was observed with nodular changes in bilateral apical region, which was later diagnosed as tuberculosis. No definite lesion was evaluated along the course of bilateral RLN and vagus nerve in rest of cases.

Fullness of ipsilateral pharyngeal wall was observed in 2 cases of unilateral VCP cases. In one case there was contralateral uvular and palatal deviation observed clinically, but in another case where CT revealed fullness of pharyngeal wall, there were no consistent signs suggestive of other cranial nerve involvement.

Table 1: Distribution of CT findings in our case series

\begin{tabular}{|c|c|c|c|c|c|c|c|c|c|c|c|c|c|c|c|c|}
\hline Sl. no. & Age & Sex & $C D$ & $U / B$ & $S$ & 1 & 2 & 3 & 4 & 5 & 6 & 7 & 8 & 9 & 10 & Other finding \\
\hline 1 & 45 & $\mathrm{M}$ & URt & $U$ & RT & + & & + & + & & & + & + & + & & \\
\hline 2 & 75 & M & U Lt & $U$ & Lt & + & & + & + & + & & + & + & & & Thyroid mass \\
\hline 3 & 75 & $M$ & U Lt & $U$ & Lt & & + & + & + & & & + & + & & & Ca pyriform sinus \\
\hline 4 & 40 & $M$ & $B$ & B & B & $\mathrm{B}+$ & $B+$ & $B+$ & $\mathrm{B}+$ & & $\mathrm{Lt}+$ & & & & & \\
\hline 5 & 57 & $\mathrm{~F}$ & U Rt & $U$ & Rt & + & + & + & + & & + & & & & & \\
\hline 6 & 62 & $\mathrm{~F}$ & U Lt & $U$ & Lt & + & + & + & + & + & & & & & & \\
\hline 7 & 75 & M & U Lt & $U$ & Lt & + & + & + & + & + & + & + & & + & & \\
\hline 8 & 58 & $M$ & U Lt & $U$ & Lt & + & + & + & + & & + & & & & & \\
\hline 9 & 72 & $M$ & U Lt & $U$ & Lt & + & + & + & + & & & & & & & \\
\hline 10 & 70 & $\mathrm{M}$ & $\mathrm{U}$ Lt & $U$ & $\mathrm{Lt}$ & + & + & + & + & + & + & & & & & $\mathrm{Ht}$ En LN and lung lesion \\
\hline
\end{tabular}

Note: Ht: Heterogeneously; En: Enhancing; LN: Lymph node; Lt: Left; Rt: Right; U: Unilateral; B: Bilateral; CD: Clinical diagnosis; S: Side; (1) Dilatation of ipsilateral pyriform sinus; (2) dilatation of ipsilateral laryngeal ventricle; (3) thickening of ipsilateral aryepiglottic fold; (4) medialization of aryepiglottic fold; (5) anteromedial displacement of arytenoids cartilage; (6) dilatation of ipsilateral vallecula; (7) fullness of vocal cord; (8) fullness of ipsilateral subglottic region; (9) fullness of ipsilateral pharyngeal wall; (10) flattening of subglottic arch 


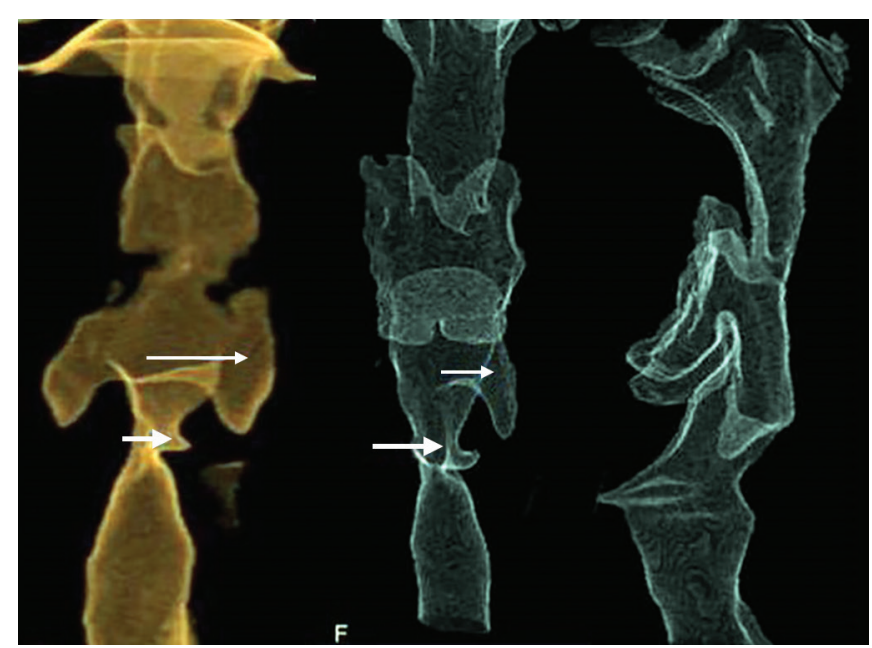

Fig. 5: Volume-rendered imaging of hypopharynx and larynx showing dilatation of left pyriform sinus (thin arrow) and laryngeal ventricle (thick arrow)

\section{DISCUSSION}

Vocal cord paralysis is a common cause of hoarseness of voice and can occur secondary to various lesions along the course of vagus nerve and its branches. The vagus nerve is the longest of the cranial nerves, extending from the brainstem to the abdomen. ${ }^{6}$ It originates from the nucleus ambiguous in the medulla of the brainstem and exits from the skull through the jugular foramen and traverses neck and chest. Also fibers from upper part of nucleus ambiguous relay with glossopharyngeal nerve and those from lower part mix up with accessory nerve forming pharyngeal plexus, which supplies pharyngeal and palatal muscles. Cranial nerves 9th, 10th, and 11th are intricately related in the medulla that all the muscles supplied by them are generally affected in medullary lesions. Hence, it is paramount to evaluate this nerve from the brainstem to the aortopulmonary window in VCP cases. Peripheral VCP is more common than a central cause, with only $10 \%$ of cases are central in origin. ${ }^{7}$ In the thorax, the left RLN is more prone to injury than the right due to its longer intrathoracic course $(12$ vs $6 \mathrm{~cm}){ }^{8}$ coming into proximity with the mediastinal surface of the left lung, passing along the mediastinal lymph nodes, and finally looping around the aortic arch. ${ }^{9}$

The course of the RLN is governed by the descent of the heart and great vessels during embryogenesis. On the right side, the recurrence of nerve loops around the subclavian artery (4th arch artery) and then ascends the neck to supply laryngeal musculature. In rare instances, if the right 4th arch development is aberrant, the right RLN does not pull down into chest and hence, runs directly from the vagus nerve to the larynx. The left RLN offshoots from the vagus as it enters the thorax. It then loops around the 6th aortic arch and ascends toward the tracheoesophageal grove. The RLN enters the larynx posterior to the cricothyroid joint and supply motor, sensory and parasympathetic innervation. The path of the RLNs may alter due to congenital vascular anomalies or anatomical distortions resulting from the presence of goiter, neoplasia, or inflammatory lesions. ${ }^{10}$

The most common iatrogenic causes of VCP are due to thyroid surgeries, carotid endarterectomy, and anterior approaches to cervical spine. ${ }^{11}$ Right RLN is more prone to surgical insult secondary to central venous catheter placement and median sternotomy. Right-side mediastinal lymphadenopathy extending cephalad to right subclavian artery region can also involve the right RLN. ${ }^{12}$ Patients with a superior sulcus tumor can show right-side VCP when the neoplasia extends into the supraclavicular space. ${ }^{13}$ The left RLN is more at risk during left upper lobectomy or cardiac surgery. ${ }^{4}$ Vocal cord palsy has been reported to be about 1.4-2.5 times more common on the left side than on the right side. ${ }^{14}$ The causes of bilateral VCP in adults can be: (1) Following thyroid surgeries, the most common reason, (2) neurogenic disorders, such as poliomyelitis, cerebrovascular accidents, multiple sclerosis, (3) neoplastic lesions of the neck and mediastinum, and (4) other causes like intubation, infection, congenital lesions, trauma, idiopathic, and bilateral neck dissection..$^{15}$ In our series of 10 patients, one case of VCP was due to thyroid mass, second was of tuberculosis, and in third case VCP was due to pyriform malignancy. In rest of 7 cases in our series, no cause of VCP could be ascertained. All the patients in our series had hoarseness of voice as chief complaint.

Although the majority of VCP cases can occur due to a known etiology, nevertheless a substantial number of cases occur without an obvious precipitating cause. The most characteristic findings of unilateral VCP are: (1) Widening of the ipsilateral laryngeal ventricle, (2) medial deviation and thickening of the aryepiglottic fold, and (3) dilatation of the pyriform sinus. ${ }^{2,16}$ The widening of laryngeal ventricle is the passive outcome of atrophy of the ipsilateral vocal cord, whereas changes in the aryepiglottic fold and the pyriform sinus occur due to paralysis of posterior cricoarytenoid muscle, the only abductor of the vocal cord. ${ }^{17}$ Atrophy of this muscle and the inability to abduct the cords culminates into anteromedial rotation of the arytenoid, medial deviation of the aryepiglottic fold, and passive dilatation of the pyriform sinus. ${ }^{17}$ In a study by Chin et al ${ }^{2}$ of 31 cases with clinically confirmed $\mathrm{VCP}$, at least two of these three $\mathrm{CT}$ findings were present in all 31 cases. In cases of $\mathrm{VCP}$, on laryngeal CT scanning, at least 10 findings have been described by Landman These include: (1) Incomplete abduction of both the true and false cords in quite breathing, (2) dilated ipsilateral laryngeal ventricle, (3) flattened ipsilateral subglottic arch, 
(4) displacement of interarytenoid notch to normal side during phonation, (5) paralyzed arytenoids cartilage anteriorly displaced and abutting or crossing the midline, (6) thinner-than-normal edge of paralyzed true vocal cord, (7) dilated ipsilateral pyriform sinus (associated with medial displacement of aryepiglottic fold), (8) dilated ipsilateral vallecula, (9) paralyzed cord lower than the normal cord during inspiration but higher than normal cord during phonation, and (10) flattened ipsilateral lateral wall of the vestibule. ${ }^{5}$ In our series, thickening and medialization of aryepiglottic folds were consistently observed in all cases.

The flattening of the subglottic arch is better appreciated on reformatted coronal images than on axial images. ${ }^{2}$ Another finding, fullness of the paralyzed cord, while analyzing it on CT, is noteworthy that it is not always recognized. The reason of failure in recognizing this in many cases may be due to failure in making precisely align the scanning plane with the true vocal cords. ${ }^{2}$

Other supportive sign of VCP on axial cuts is anteromedial displacement of the ipsilateral arytenoids cartilage with medial displacement of the posterior vocal cord margin. These twin features, medialization of the posterior vocal cord margin and ipsilateral ventricular distension, result in the residual airway taking a shape identical to a ship's sail, commonly known as the "sail" $\operatorname{sign}^{18}$ (Fig. 6). Oblique imaging may lead to distorted appearance of VCP at the level of the true vocal cords with anterior subglottic air mimicking an air distended laryngeal ventricle. In the setting of true VCP, oblique axial imaging obtained at level of cords may also show

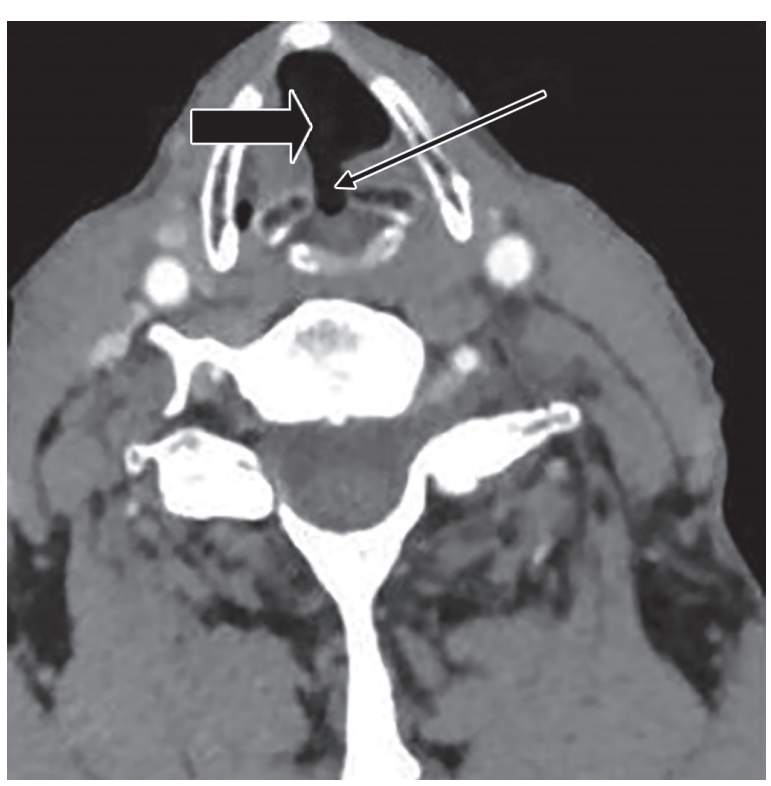

Fig. 6: Axial CT scan obtained at the level of the vocal cords during quiet respiration shows anteromedially displaced ipsilateral aryepiglottic cartilage and the related medial position of the posterior vocal cord margin ( thin arrow), as well as dilatation of ipsilateral laryngeal ventricle (thick arrow) giving sail's sign subglottic air anteriorly, opposite to a dilated laryngeal ventricle on side of VCP, giving it a mushroom-like appearance (Fig. 7). This sign should be considered as a supportive sign of unilateral VCP on oblique axial CT scan done at the level of true vocal cords. ${ }^{4}$

While evaluating, we should be aware of various mimics of VCP that can lead to misdiagnosis and hence, unnecessary investigations and inappropriate management might lead to poor prognosis. First, laryngeal and pyriform sinus squamous cell carcinoma can mimic VCP by encroaching the aryepiglottic fold, resulting in immobility or thickening of vocal cord. ${ }^{4}$ In our series, one case of VCP had a pyriform growth which was later confirmed histologically. Second, medial dislocation or subluxation of arytenoids cartilage can be labeled as VCP. It usually occurs after difficult and traumatic intubation or due to blunt laryngeal trauma. ${ }^{4}$ Third, tilted patient positioning or the use of oblique imaging planes can result into asymmetric visualization of the true vocal cords. On axial imaging, this asymmetry can simulate VCP when normal subglottic air present anterior to an obliquely imaged vocal cord looks like a dilated ventricle. Imaging in the same plane of cricoarytenoid joint on the same axial or oblique axial reformatted image can solve this problem. ${ }^{4}$ Apart from these fallacies, it is pertinent to correctly appreciate the laryngeal appearance after surgical medialization or reinnervation for the treatment of VCP. ${ }^{4}$ Another perplexing condition is bilateral VCP, where characteristic findings of VCP are present on both sides, making it difficult to correctly identify. ${ }^{17}$ In our

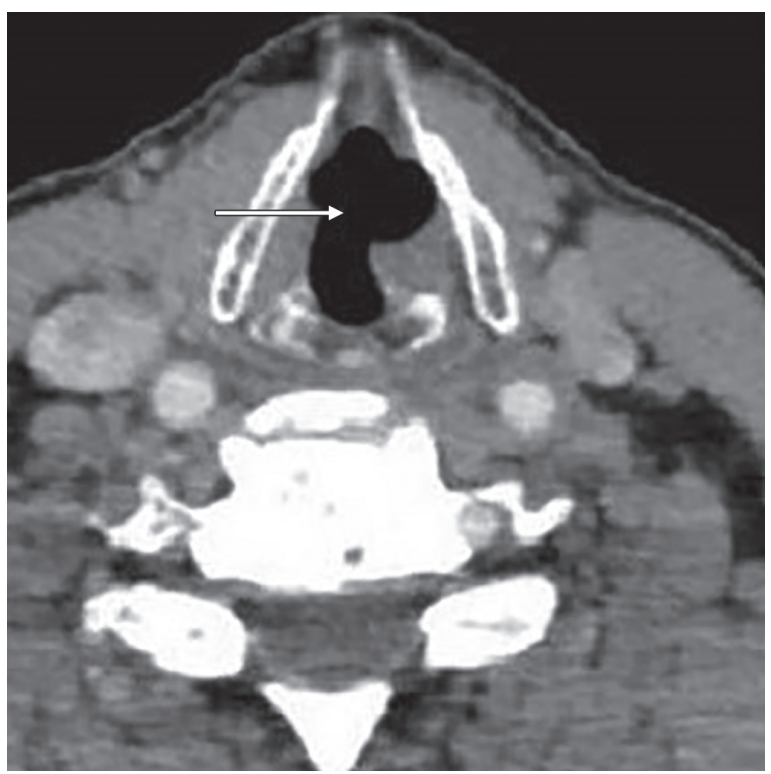

Fig. 7: A patient with left VCP. On contrast-enhanced axial CT scan at level of the vocal cords shows ipsilateral laryngeal ventricle dilatation and posterior cord medialization with anterior contralateral subglottic air giving mushroom-like appearance 
series, one case of bilateral VCP revealed dilatation of bilateral pyriform sinus, dilatation of bilateral laryngeal ventricle, thickening of bilateral aryepiglottic fold, medialization of bilateral aryepiglottic fold, and dilatation of unilateral vallecula.

The differential diagnosis, clinical presentation, and management differ for patients having a high vagal lesion or central neuropathy and those with a low vagal paralysis. Clinically, features that can help to localize the level of vagal nerve lesion are features due to multiple cranial nerve involvement, and appearance due to their involvement can be best appreciated on CT scanning. On CT scanning, outward bowing of the oropharyngeal and hypopharyngeal contour, thinning of the constrictor muscle ${ }^{2}$ uvula deviation to normal side ${ }^{4}$ are in accord with ipsilateral pharyngeal plexus injury. These findings localize the lesion to a level either just below the skull base or more cranially in the brainstem. ${ }^{2}$ The chance of developing an isolated laryngeal motor nerve involvement encased by an intracranial lesion is highly improbable due to the close relation of the corticobulbar fibers to the surrounding structures. ${ }^{12}$ If central-proximal lesion is suspected, further evaluation with magnetic resonance imaging (MRI) of the posterior cranial fossa is warranted. ${ }^{4}$

Cranial neurologic lesions account for a relatively small percentage of all laryngeal paralyses. They may be congenital, secondary to trauma, infection, neoplasm, or vascular accident. Depending on the relationship of lesion to the nucleus ambiguous, these can be further divided as supranuclear, nuclear, or infranuclear. Supranuclear lesions due to massive insult to the cortex usually present as an upper motor neuron lesion and result in bilateral spastic laryngeal paralysis. Nuclear lesion is a bulbar or lower motor neuron type of palsy and usually associated with involvement of other nuclei in medulla. Infranuclear lesion may be located at or near the jugular foramen and are associated with other lower cranial nerve palsies. Whereas, peripheral neurologic lesions comprise any or all possible combination of paralysis of the superior laryngeal nerve (SLN), RLN, or both. ${ }^{19}$

In certain situations, an additional chest CT scan is advisable when the neck scan shows an enlarged aortopulmonary window, supraclavicular nodes, or right highest mediastinal nodes without an obvious primary malignant lesion, such as mid to low esophageal cancer or lung cancers that are beyond the confines of the neck scan. ${ }^{12}$ A negative chest and neck CT scan confirms clinicians that VCP is essentially idiopathic in origin, hence, avoiding the need for aggressive diagnostic testing and therapeutic interventions, both. ${ }^{13}$

Albeit a CT scan is important, the role of proper history taking cannot be ignored. This is particularly important in conditions: (1) VCP in a patient during follow-up for a known malignancy, as it may signal recurrence or aggravation, (2) VCP after tracheostomy, chemoport insertion, or any surgery of lung, esophagus indicating iatrogenic paralysis, and (3) a 10 to 30-year history of VCP, indicating chronic nature of lesion, i.e., tuberculosis. ${ }^{12}$

\section{CONCLUSION}

The appreciation of characteristic radiological signs, as well as the knowledge of the normal anatomy, its variations, and the diseases affecting the vagus nerve play an important role in choosing the best imaging modality to be used. This study is helpful in reliably suggesting the presence of VCP on routine CT in those cases who present without apparent clinical history and clinical features and localizing the level of vagal lesion, eventually directing us to specific management of a particular lesion. Obviously, in the absence of history of clinical complaints, we can reliably predict the presence of VCP by recognizing specific signs on CT scan. Correctly identifying the cause of VCP can help clinicians as well as patients in early detection and management of a neoplastic lesion, in detecting recurrence or aggravation of primary tumor, or in dissemination of malignancy.

\section{REFERENCES}

1. Richardson BE, Bastian RW. Clinical evaluation of vocal fold paralysis. Otolaryngol Clin North Am 2004 Feb;37(1): 45-58.

2. Chin SC, Edelstein S, Chen CY, Som PM. Using CT to localize side and level of vocal cord paralysis. AJR Am J Roentgenol 2003 Apr;180(4):1165-1170.

3. Collazo-Clavell ML, Gharib H, Maragos NE. Relationship between vocal cord paralysis and benign thyroid disease. Head Neck 1995 Jan-Feb;17(1):24-30.

4. Paquette CM, Manos DC, Psooy BJ. Unilateral vocal cord paralysis: a review of CT findings, mediastinal causes, and the course of the recurrent laryngeal nerves. Radiographics 2012 May-Jun;32(3):721-740.

5. Landman GH. Laryngography: cine laryngography. Baltimore (MD): Williams \& Wilkins; 1970. p. 62-75.

6. Curtin HD. The larynx. In: Som PM, Curtin HD, editors. Head and neck imaging. 4th ed. St. Louis (MO): Mosby; 2003. p. 1601-1603.

7. Levine HL, Tucker HM. Surgical management of the paralyzed larynx. In: Bailey BJ, Biller HF, editors. Surgery of the larynx. Philadelphia (PA): Saunders; 1985. p. 117-134.

8. Yumoto E, Minoda R, Hyodo M, Yamagata T. Causes of recurrent laryngeal paralysis. Auris Nasus Larynx 2002 Jan;29(1): 41-45.

9. Titche LL. Causes of recurrent laryngeal nerve paralysis. Arch Otolaryngol 1976 May;102(5):259-261.

10. Garcia MM, Magalhaes FP, Dadalto GB, Moura MV. Imaging evaluation of vocal cord paralysis. Radiol Bras 2009;42(5): 321-326. 
11. Rosenthal LH, Benninger MS, Deeb RH. Vocal fold immobility: a longitudinal analysis of etiology over 20 years. Laryngoscope 2007 Oct;117(10):1864-1870.

12. Song SW, Jun BC, Cho KJ, Lee S, Kim YJ, Park SH. CT evaluation of vocal cord paralysis due to thoracic diseases: a 10-year retrospective study. Yonsei Med J 2011;52(5):831-837.

13. Glazer HS, Aronberg DJ, Lee JK, Sagel SS. Extralaryngeal causes of vocal cord paralysis: CT evaluation. AJR Am J Roentgenol 1983 Sep;141(3):527-531.

14. Furukawa M, Furukawa MK, Ooishi K. Statistical analysis of malignant tumours detected as the cause of vocal cord paralysis. ORL J Otorhinolaryngol Relat Spec 1994 MayJun;56(3):161-165.
15. Holinger LD, Holinger PC, Holinger PH. Etiology of bilateral abductor vocal cord paralysis: a review of 389 cases. Ann Otol Rhinol Laryngol 1976 Jul-Aug;85(4 Pt 1):428-436.

16. Robinson S, Pikaranta A. Radiology findings in adult patients with vocal fold paralysis. Clin Radiol 2006 Oct;61(10):863-867.

17. Dankbaar JW, Pameijer FA. Vocal cord paralysis: anatomy, imaging and pathology. Insights Imaging 2014 Dec;5(6): 743-751.

18. Agha FP. Rcurrent laryngeal nerve paralysis: a laryngographic and computed tomographic study. Radiology 1983 Jul;148(1):149-155.

19. Tucker HM. Neurologic disorders. In: Tucker HM, editor. The Larynx. 2nd ed. New York (NY): Thieme; 1993. p. 247-249. 